\title{
TOLERANCES IN X-BAND MAIN LINACS OF FUTURE LINEAR COLLIDERS
}

\author{
T. Higo, K. Kubo and K. Yokoya, KEK, Tsukuba, Japan
}

\begin{abstract}
Tracking simulations have been performed for a realistic design of main linacs of X-band linear collider. We discuss about choice of the phase of the accelerating field relative to the bunches. The tolerances of the phase and the amplitude errors are studied.
\end{abstract}

\section{INTRODUCTION}

In order to preserve the low emittance through the main linacs of future linear colliders, various effects should be considered, errors of injection conditions, RF accelerating field, strength of magnets, misalignment and mechanical vibrations of magnets and accelerating structures and so on. Tracking simulations have been performed for a realistic design of main linacs of X-band linear collider with these errors [1]. Some of the estimated tolerances based on the simulations have been reported before [2,3]. Here we discuss about the choice of the phases of the accelerating field relative to the bunches and the tolerances of the phase and the amplitude errors. Because the vertical emittance will be much smaller than the horizontal emittance and tolerances will be much tighter, simulation results were shown only in the vertical plane.

\section{PARAMETERS OF THE LINAC}

One possible parameter set of the main linac of future linear colliders with CMS energy of $1 \mathrm{TeV}$ was used in the simulations. Important parameters are listed in Table 1. The optics is a series of FODO cells, and the phase advance per length is proportional to the inverse of the square root of the beam energy $[2,4]$.

Table 1: Parameters of the linac.

\begin{tabular}{|c|c|}
\hline Initial beam energy & $10 \mathrm{GeV}$ \\
\hline Final beam energy & $500 \mathrm{GeV}$ \\
\hline Bunch charge & $1.01 \times 1010$ \\
\hline Bunch length & $125 \mathrm{~mm}$ \\
\hline $\begin{array}{c}\text { Nominal normalized vertical } \\
\text { emittance }\end{array}$ & $3 \times 10-8 \mathrm{~m}-\mathrm{rad}$ \\
\hline Frequency of RF & $11.424 \mathrm{GHz}$ \\
\hline Acc. gradient with loading & $56.7 \mathrm{MV} / \mathrm{m}$ \\
\hline Number of acc. structures & $4788 / \mathrm{linac}$ \\
\hline Acc. structure length & $1.8 \mathrm{~m}$ \\
\hline Averaged structure iris & $0.18 \lambda$ \\
\hline Length of a FODO cell & $8.6 \mathrm{~m}$ at $10 \mathrm{GeV}$ \\
\hline Phase advance/ FODO cell & $90 \mathrm{o}$ at $10 \mathrm{GeV}$ \\
\hline
\end{tabular}

\section{CHOICE OF RF PHASE}

Energy distribution in a bunch is important in considering emittance preservation, e.g. as known as 'BNS damping'
[5], and it can be controlled by the choice of the phases of the RF field along the linac. In addition to the relative energy spread $\left(\sigma_{E} / E\right)$, let us define another parameter 'energy-z correlation' as follows,

$$
\delta E / E \equiv \frac{1}{E \sigma_{z} q} \int E(z) z \rho(z) d z
$$

where $\sigma_{z}$ is the bunch length, $q$ the total charge, $z$ the longitudinal coordinate, $\rho(z)$ the charge density and $E(z)$ the average energy at $z$.
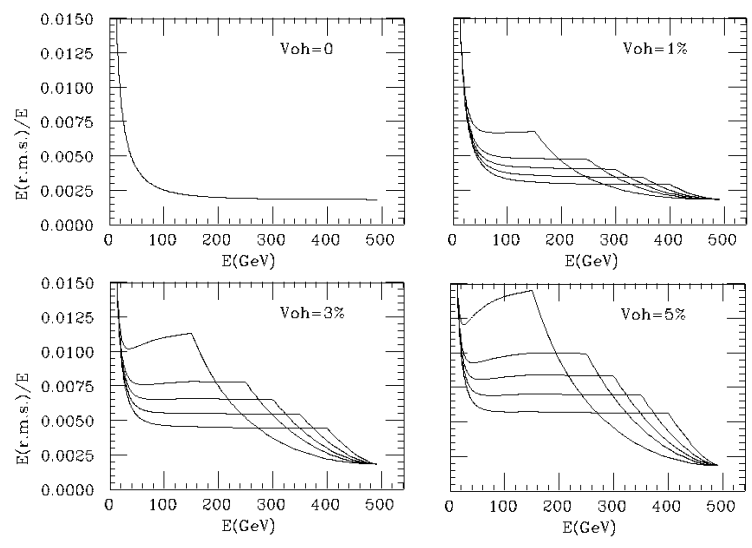

Figure 1: Relative energy spread along the linac.

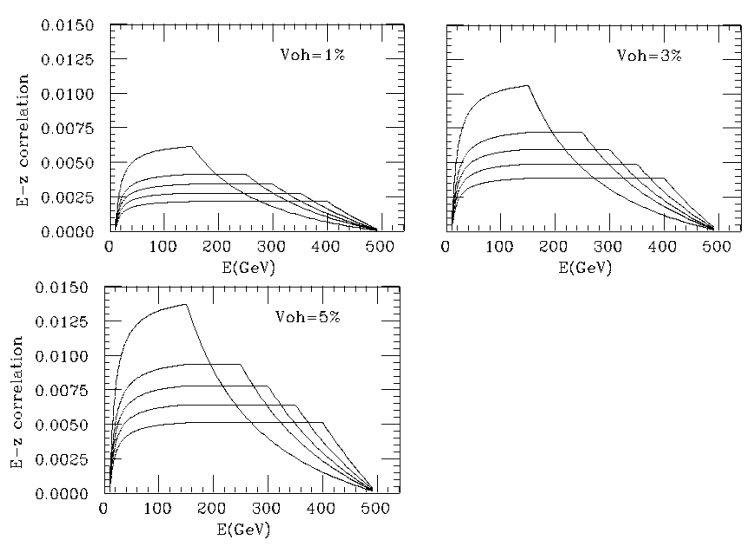

Figure 2: 'Energy-z correlation' along the linac.

We simulated six cases : (a) Constant phase, (b) Change phases at $150 \mathrm{GeV}$, (c) Change phases at $150 \mathrm{GeV}$ and $250 \mathrm{GeV}$, (d) at $150 \mathrm{GeV}$ and $300 \mathrm{GeV}$, (e) at $150 \mathrm{GeV}$ and $350 \mathrm{GeV}$, (f) at $150 \mathrm{GeV}$ and $400 \mathrm{GeV}$. In all cases, phases are chosen to make $\delta E$ at the end of linac zero, and $\sigma_{E}$ minimum. The phase in the case (a) is decided by this constraint as $15.4 \mathrm{o}$ (off crest angle of the bunch center). In the case of (c) (f), the phases are chosen to make $\delta E$ constant in the center energy region. 'Over head 
voltage', $V_{o h}$, was a parameter which decide the phases in the cases (b) (e).

$$
V_{o h} \equiv V / V_{(a)}-1
$$

where $V$ is total of the RF voltage and $V_{(a)}$ that in the case (a). Making $\delta E$ the bigger, $\delta E$ becomes bigger and more voltage is needed for the same beam energy.

Cases of $V_{o h}=0,1,3$ and $5 \%$ were simulated $\left(V_{o h}=0\right.$ means the case (a)). $\sigma_{E} / E$ is shown in Fig. 1 for the all cases and $\delta E / E$ is shown in Fig. 2 for the all cases except (a) which has almost zero $\delta E / E . \delta E / E=0$ and $\sigma_{E} / E=0.02$ were assumed at the beginning.

$\delta E / E$ for the auto phasing condition is approximately constant along the linac and a rough estimation gives

$$
\delta E / E \text { (auto phasing) } \approx e q \beta^{2} W_{T}\left(2 \sigma_{z}\right) / 8 E \approx 0.022
$$

This is bigger than that even in the $V_{o h}=5 \%$ cases. To make such big energy slope, a big over head voltage is needed and probably will not be realistic.

Emittance dilutions were simulated for injection errors, vibration of quadrupole magnets and misalignment of quadrupole magnets with a simple orbit corrections.

We take 'effective emittance' $\left(\varepsilon_{\text {eff }}\right)$ as a measure of the beam quality for discussion of the jitters or fast errors which will not be corrected and emittance $(\varepsilon)$ for the fixed or slowly changing errors which will be corrected.

$$
\begin{gathered}
\varepsilon_{\text {eff }} \equiv \sqrt{\left\langle y^{2}\right\rangle\left\langle y^{\prime 2}\right\rangle-\left\langle y y^{\prime}\right\rangle^{2}} \\
\varepsilon \equiv \sqrt{\left\langle(y-\langle y\rangle)^{2}\right\rangle\left\langle\left(y^{\prime}-\left\langle y^{\prime}\right\rangle\right)^{2}\right\rangle-\left(\left\langle y y^{\prime}\right\rangle-\langle y\rangle\left\langle y^{\prime}\right\rangle\right)^{2}}
\end{gathered}
$$

where \langle\rangle means the average of all particles.

Fig.3(a) shows $\varepsilon_{\text {eff }}$ dilution due to injection jitters. The offset and the angle of the injected beam were randomly set as gaussian with sigma of 0.5 of the beam size. Except for the injection error, the linac was assumed to be perfect. Fig.3(b) shows $\varepsilon_{\text {eff }}$ dilution due to transverse vibrations of quadrupole magnets. The transverse offset of quads were randomly set as gaussian with sigma of $10 \mathrm{~nm}$. Except for the injection error, the linac was assumed to be perfect. The averages over 200 random seeds are shown.
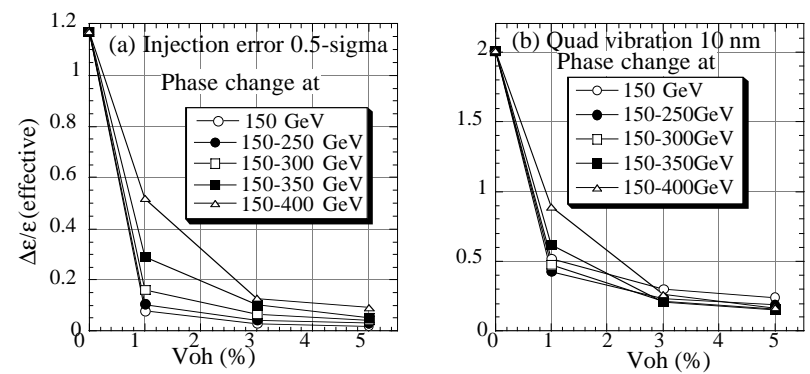

Fig. 3: Effective emittance dilution vs. $V_{\text {oh }}$ with (a) injection jitters and (b) vibration of quadrupole magnets.
Fig.4 shows $\varepsilon$ dilution due to transverse misalignment of quadrupole magnets with orbit corrections. A simple one-to-one orbit correction was assumed. There were steering magnets and BPMs at all quadrupoles. Only BPMs at focusing quads were used and the beam is steered to make the BPM readings zero. Transverse offset of the quads were randomly set as gaussian with sigma of 2 micron. The BPMs also have random transverse offset with sigma of 2 micron with respect to the quads' center. Resolution of the BPMs was 1 micron. Accelerating structures were aligned perfectly w.r.t. the beam. The averages over 100 random seeds are shown.

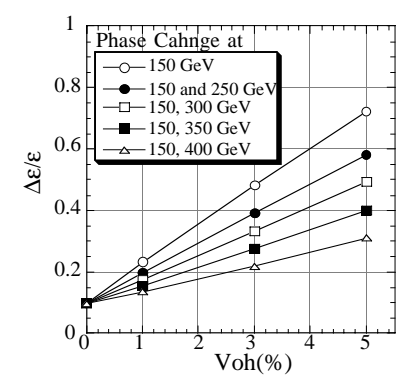

Fig. 4 : Average emittance dilution due to misalignment of quadrupole magnets with orbit correction.

It is obvious that $\delta E / E$ should be large, which means large over head voltage, to suppress the emittance dilution caused by the injection error and the quads' vibrations. On the other hand, a large energy spread makes the dispersive effects large which is shown in Fig.4. Of cause, considering the efficiency, a too large over head voltage should be avoided. As conclusion, the phases should be decided as a result of some compromise and the optimum $V_{o h}$ will be 3 5\%.

For further simulations we pick up the four cases $V_{\text {oh }}=0, V_{\text {oh }}=1 \%$ phase changes at $150 \mathrm{GeV}$ and $250 \mathrm{GeV}$, $V_{\text {oh }}=3 \%$ phase changes at $150 \mathrm{GeV}$ and $300 \mathrm{GeV}$ and $V_{\text {oh }}=5 \%$ phase changes at $150 \mathrm{GeV}$ and $350 \mathrm{GeV}$.

\section{RF JITTER TOLERANCE}

Simulations with jitter of the RF amplitude and phase have been done for the four cases of the phase choice. In the simulations, considering DLDS scheme [6], one RF unit consists of 12 accelerating structures and each unit has the same error of amplitude and phase with Gaussian distributions. All RF units have the same r.m.s. of the errors and no correlation was considered between different units.

\subsection{Beam Energy}

Here, we looked at final beam energy and energy spread with the error of the RF.

Amplitude jitter causes pulse to pulse fluctuations of the beam energy (average of the particles in one pulse). Assuming independent errors, the r.m.s. of the relative error of the beam energy can be estimated simply as 


$$
\sigma_{\langle E\rangle} / E=\sigma_{a m p} / \sqrt{N_{R F}}=\sigma_{a m p} / \sqrt{399}
$$

where $\sigma_{a m p}$ is the r.m.s. of the relative amplitude jitter of each unit and $N_{R F}=399$ the number of RF units. Simulations confirmed the equation (1) and showed that the pulse to pulse fluctuation of the energy spread will be less than $1 \times 10^{-6}$ up to $\sigma_{a m p}=8 \%$.

The phase jitter causes the pulse to pulse fluctuations of the beam energy and also reduces the mean beam energy. Results of simulations with 200 random seeds are shown in Fig.5, the average of the relative beam energy and the fluctuations of the beam energy vs. r.m.s. of the phase jitter.
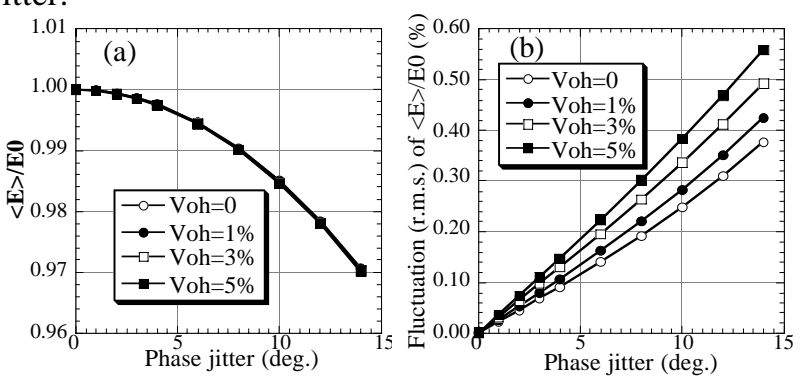

Figure 5: (a) Average of the relative beam energy and (b) fluctuations of the beam energy vs. RF phase jitter.

Assuming that the tolerance of the final energy error due to the RF error is $0.1 \%$, the tolerances for the RF amplitude and phase jitters will be about $2 \%$ and 30 , respectively.

\subsection{Transverse motion}

Energy error along the linac may affect the transverse motion of the beam. The pulse to pule energy difference will cause a pulse to pule orbit difference due to the dispersive effect.

As the initial condition, we set random misalignment of the quads and calculated strength of the steering magnets for orbit corrections without RF errors. The misalignment and the setting of the magnets were saved and used in simulations with RF errors. Effective emittances were calculated with the position and the angle without jitters as the reference.

Fig. 6 shows $\varepsilon_{\text {eff }}$ dilution vs. r.m.s. of the amplitude jitter and the phase jitter for the four cases of the phase choice. Each point represents average of 200 random seeds for jitters in one initial condition (1 linac). The same initial condition was used in the same case of the phase choice. Because the results are statistically poor, simulations were done for 50 different initial conditions and 50 different RF errors for each initial condition (50 linacs, 50 pulses for each linac) only in the case of $V_{\text {oh }}=3 \%$. Fig. 7 shows $\varepsilon_{\text {eff }}$ increase vs. r.m.s. of the amplitude and phase jitter. In this figure, the average and the r.m.s. fluctuation of the additional increase of $\varepsilon_{\text {eff }}$ due to the jitters are shown.
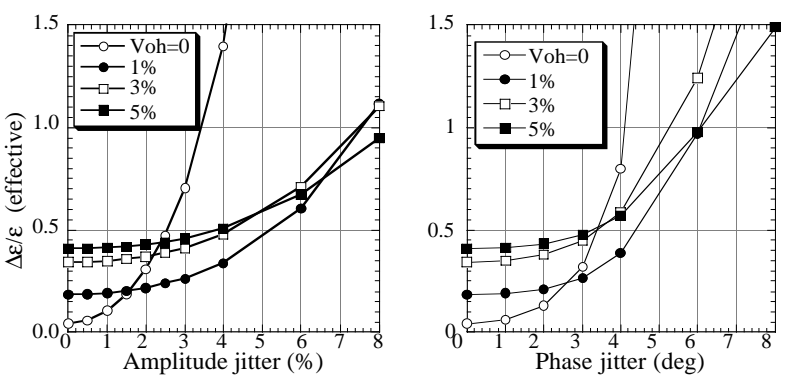

Fig.6: Effective emittance vs. r.m.s. of (a) amplitude jitter and (b) phase jitter. Each point represents average of 200 random seeds for jitters in one initial condition.
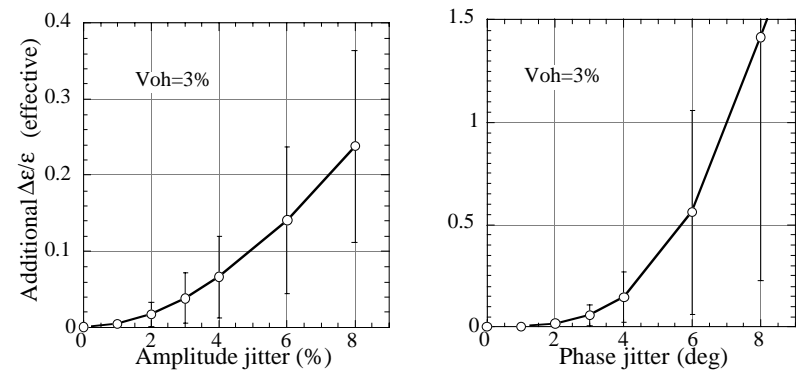

Fig. 7: Increase of effective emittance vs. r.m.s. of (a) amplitude jitter and (b) phase jitter of RF. Average and r.m.s. fluctuation from 50 different misalignments and 50 different jitters are shown.

Compared with the effect to the final energy, the effect of the RF jitter to the emittance will be not significant.

\section{SUMMARY}

The choice of the phases and the tolerances of the jitters of the phase and the amplitude of the RF accelerating fields have been studied for future X-band linear colliders. The phase choice can be represented by the 'over head voltage' $\left(V_{o h}\right)$ and its optimum will be $3 \sim 5 \%$. The tolerances of the jitters will be decided by the effects to the final energy error not to the emittances. Requiring the error of the final energy of less than $0.1 \%$, the jitter tolerances will be about $2 \%$ for the amplitude and about 3o for the phase.

\section{ACKNOWLEDGMENT}

The authors would like to thank Dr. T.O.Raubenheimer of SLAC for valuable suggestions and discussions.

\section{REFERENCES}

[1] K.Kubo et. al., NLCNote-13 (1995).

[2] JLC Design Study Group, KEK-Report, 97-1, (1997).

[3] e.g., K.Kubo et.al., Proc. of PAC95, p3112, (1995).

[4] "http://www.slac.stanford.edu/xorg/ilc-trc/Table3_2/table3_2.html".

[5] V.Balakin, A.Novokhatsky and V.Smirnov, Proc. of HEACC83, p119 (1983)

[6] H.Mizuno and Y.Otake, Proc. of LINAC94, p463, (1994) 